Kamm Jehli, Sandra; Jaumann, Johannes

\title{
Auf den Lernspuren eines europäischen Lernwerkstätten-Treffens. Hintergründe, konzeptionelle Überlegungen, Grenzen, Irritationen
}

Hildebrandt, Elke [Hrsg.]; Peschel, Markus [Hrsg.]; Weißhaupt, Mark [Hrsg.]: Lernen zwischen freiem und instruiertem Tätigsein. Bad Heilbrunn : Verlag Julius Klinkhardt 2014, S. 174-187. - (Lernen und Studieren in Lernwerkstätten)

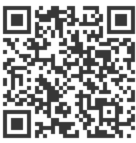

\section{Quellenangabe/ Reference:}

Kamm Jehli, Sandra; Jaumann, Johannes: Auf den Lernspuren eines europäischen Lernwerkstätten-Treffens. Hintergründe, konzeptionelle Überlegungen, Grenzen, Irritationen - In: Hildebrandt, Elke [Hrsg.]; Peschel, Markus [Hrsg.]; Weißhaupt, Mark [Hrsg.]: Lernen zwischen freiem und instruiertem Tätigsein. Bad Heilbrunn : Verlag Julius Klinkhardt 2014, S. 174-187 - URN: urn:nbn:de:0111-pedocs-193461 - DOI: 10.25656/01:19346

https://nbn-resolving.org/urn:nbn:de:0111-pedocs-193461

https://doi.org/10.25656/01:19346

in Kooperation mit / in cooperation with:

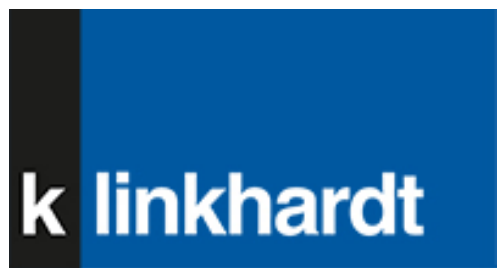

http://www.klinkhardt.de

\section{Nutzungsbedingungen}

Gewährt wird ein nicht exklusives, nicht übertragbares, persönliches und beschränktes Recht auf Nutzung dieses Dokuments. Dieses Dokument ist ausschließlich für den persönlichen, nicht-kommerziellen Gebrauch bestimmt. Die Nutzung stellt keine Übertragung des Eigentumsrechts an diesem Dokument dar und gilt vorbehaltlich der folgenden Einschränkungen: Auf sämtlichen Kopien dieses Dokuments müssen alle Urheberrechtshinweise und sonstigen Hinweise auf gesetzlichen Schutz beibehalten werden. Sie dürfen dieses Dokument nicht in irgendeiner Weise abändern, noch dürfen Sie dieses Dokument für öffentliche oder kommerzielle Zwecke vervielfältigen, öffentlich ausstellen, aufführen, vertreiben oder anderweitig nutzen.

Mit der Verwendung dieses Dokuments erkennen Sie die Nutzungsbedingungen an.

\section{Terms of use}

We grant a non-exclusive, non-transferable, individual and limited right to using this document.

This document is solely intended for your personal, non-commercial use. Use of this document does not include any transfer of property rights and it is conditional to the following limitations: All of the copies of this documents must retain all copyright information and other information regarding legal protection. You are not allowed to alter this document in any way, to copy it for public or commercial purposes, to exhibit the document in public, to perform, distribute or otherwise use the document in public.

By using this particular document, you accept the above-stated conditions of use.

\section{Kontakt / Contact:}

\section{peDOCS}

DIPF | Leibniz-Institut für Bildungsforschung und Bildungsinformation Informationszentrum (IZ) Bildung

E-Mail: pedocs@dipf.de

Internet: www.pedocs.de

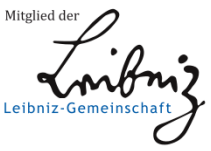




\section{Auf den Lernspuren eines \\ europäischen Lernwerkstätten-Treffens - \\ Hintergründe, konzeptionelle Überlegungen, Grenzen, Irritationen}

Im Oktober 2012 fand die europäische Lernwerkstätten-Tagung in Waldmünchen im Bayerischen Wald zum Thema „Grenzen“ statt. Dieser Beitrag befasst sich mit den konzeptionellen Überlegungen dieses Treffens und möchte eine Verbindung schaffen zwischen dem Treffen der Lernwerkstätten an Hochschulen und dem alljährlichen europäischen Lernwerkstätten-Treffen.

Die Tagung bot viel Raum für neue und ungewöhnliche Wege, wie beispielsweise die Gestaltung einer Lernumgebung ohne Textelemente. Ziel der Organisatoren war es, mit möglichst wenig Instruktion motivierende Denkanstöße zu liefern. Bei den Vorüberlegungen kamen einige Fragen auf, die man unter folgendem Gedanken subsumieren kann: „Wie können wir uns frei machen von unserer starren Haltung zu den Dingen, um eine Lernumgebung zu arrangieren, die der Heterogenität des menschlichen Denkens gerecht wird und den Zugang zum Thema nicht im Vorhinein eingrenzt?".

„Es ist die wichtigste Kunst des Lehrers, die Freude am Schaffen und am Erkennen zu erwecken." (Einstein 1986, 25).

\section{Gedanken zu Beginn}

Blickt man auf die Anfänge der Lernwerkstatt-Entwicklung zurück, waren Lernwerkstätten Orte, an denen „Lehrerinnen und Lehrer aktiv, forschend entdeckend kreativ und offen lernen sollten, um mit Kindern auf andere neue Weise Schule machen zu können" (Wedekind 2006). Durch sein Setting besinnt sich die Tagung auf ihre Wurzeln, wie sie schon 1988 von Karin Ernst (1988) beschrieben wurden und schenkt dadurch den Lernenden einen Raum, in dem sie sich „Raum und Zeit nehmen können, für ein beziehungsvolles Lernen zwischen Sachauseinandersetzung und Selbstfindung" (Hagstedt 2008, 1). 
Durch die Zusammenkunft von Pädagogen aus dem deutschsprachigen Raum entsteht ein Ort der Reflexion des eigenen Tuns in der eigenen Lernwerkstattarbeit und der wechselseitigen Inspiration. Die Teilnehmer des europäischen Lernwerkstätten-Treffens waren alle Experten, die selbst Lernwerkstätten leiten und in ihnen arbeiten. An der Tagung wurden sie wieder zu Lernenden, die sich aufmachten neue Lernwege einzuschlagen und abschließend ihre Erfahrungen und die daraus folgenden Erkenntnisse für die Praxis im Plenum zu diskutieren. Für viele Teilnehmer ist die Tagung auch Impulsgeber und Auftankstelle für die Umsetzung in der eigenen Arbeit. Die Teilnehmer erhalten durch die Tagung somit die Chance ihre eigene konstruktivistische Sicht des Lernens zu hinterfragen und können dadurch den möglicherweise starren alltäglichen Auffassungen von Lehren und Lernen im schulischen Kontext entkommen. Es gilt sich also immer wieder auf seine Wurzeln zu besinnen und sich unvoreingenommen auf eine neue Lernumgebung einzulassen.

Während der Lehrerbildung erfahren die Studierenden in den Lernwerkstätten der Hochschulen eine offene, konstruktivistische Form des Lernens als Lernende. In ihrer späteren Rolle als Lehrperson befinden sie sich oft in einer Alltagsumgebung, in der die Instruktion eine große Rolle spielt. Oft ergeben sich diverse Hindernisse beim Transfer der erlebten Erfahrungen in den Schulalltag. Während die Tagungen der Lernwerkstätten an Hochschulen besonders einen forschenden Blick auf die Entwicklung der Hochschullernwerkstätten legen, werfen die internationalen Lernwerkstätten-Tagungen ihren Blick mehr auf die alltägliche Praxis von Lernwerkstätten an Schulen. Hierzu begeben sich die Teilnehmer wieder in die Rolle der Lernenden um nachvollziehen zu können, welche Entwicklung die Schüler auf ihren Lernwegen durchschreiten, um dies für sich zu analysieren und später in ihre Praxis zu integrieren.

\section{Struktur der Lernwerkstätten-Tagung in Waldmünchen}

\subsection{Organisation}

Für die Organisation der Tagung bekam eine Gruppe von jungen Lernwerkstättlern aus der Lernwerkstätten-Community, darunter auch die Autoren dieses Artikels, die Chance und das Vertrauen der Erfahrenen, die Tagung zu organisieren und für sie Neues auszuprobieren. Durch die spezielle geografische Zusammensetzung des Teams, deren Mitglieder sich über ganz Deutschland und die Schweiz verteilen, mussten neue Wege der Kommunikation während den Vorbereitungen zur Tagung gegangen werden. Es wurden Skype-Konferenzen abgehalten, der Datenaustausch fand über Cloud-Software statt. Begleitend dazu wurde der Prozess mit einem webbasierten Editor zur kollaborativen Bearbeitung von Texten festgehalten. Dies ermöglichte den zeit- und ortsunabhängigen Zugriff auf die Daten und Einsicht in die Arbeitsprozesse der Anderen. Die interessanten Erfahrungen 
im Hinblick auf die neuen Medien wurden in die Tagung mit einbezogen. Zum Beispiel wurden für die Tagungsdokumentation hauptsächlich Tablet-PCs verwendet, mit denen die Teilnehmer die Möglichkeit hatten, ihre Stimmungen in einer Audio-Datei festzuhalten oder ein kurzes Video zu drehen. Des Weiteren bestand die Möglichkeit sich über PiratePad auszutauschen und Statements über die Tagung abzugeben. Der Einsatz neuer Medien stellte einen Versuch dar, sich diesen anzunähern und die Eignung für die Lernwerkstattarbeit auszutesten.

Das Barcamp als Abendveranstaltung machte es sich zum Ziel, Erfahrungen untereinander auszutauschen. Ein Barcamp ist eine offene Veranstaltung mit Vorträgen und Workshops. Die Inhalte und Themen werden von den Teilnehmern zu Beginn des Barcamps entwickelt und vorgestellt. Die Teilnehmer profitieren von dem großen Erfahrungsschatz der anderen Teilnehmer. Während der WorkshopPhase können beispielsweise Projekte, Konzepte oder Ideen vorgestellt werden und im Anschluss zur Diskussion gebracht werden. So wurde beispielsweise ein Workshop zum Thema Lernwerkstattarbeit für Neulinge angeboten, ebenso wie ein Vortrag zum Thema Lernwerkstatt-Erfahrungen über 20 Jahre. Gleichfalls gut besucht wurden der Vortrag eines Schweizer Grenzgedichtes in Mundart und ein Workshop über geführtes Malen. Bei dieser Form der Veranstaltung wird deutlich, welche vielfältigen Ressourcen die einzelnen Teilnehmer zur jährlichen Lernwerkstätten-Tagung mitbringen und wie man diese gewinnbringend in einer Tagung zur Geltung bringen kann.

\subsection{Ort - Thema}

\begin{tabular}{|l|l|}
\hline Toll: An den Grenzsteinen und \\
Grenzpfosten (ohne Stacheldraht) \\
hin- und herzugehen - Inland- Aus- \\
land in Sekundensprüngen“
\end{tabular}

Abb. 1: Grenzen

Als Veranstaltungsort wurde Waldmünchen im bayerischen Wald gewählt. Die Tagung und die Unterbringung erfolgten vorwiegend in der Jugendbildungsstätte Waldmünchen, wodurch auch nach den offiziellen Arbeitsphasen ein reger Aus- 
tausch stattfand. Der Ort Waldmünchen inspirierte zum Thema „Grenzen“ unter anderem durch die nahegelegene Grenzlinie zwischen Deutschland und Tschechien, die 1990 nach dem Fall des Eisernen Vorhangs wieder geöffnet wurde, die Zeitzeugnisse und seine Zeitzeugen. Weitere Grenzerfahrungen in der Nähe (Hochseilgarten, Wünschelrutengänger, Eisvogelsteig) wurden als Wahlangebote ausfindig gemacht und weckten unter den Teilnehmern reges Interesse. Weitere Impulse zum Thema "Grenze" bekamen die Teilnehmer durch täglich wechselnde Zitate und Sprüche an den Zimmertüren.

„Es geht nicht darum, Grenzen zu verschieben, sondern ihnen den trennenden Charakter für die Menschen zu nehmen." (Weizsäcker 2001, 51).

$\mathrm{Ob}$ es die eigenen Grenzen, das Überwinden mathematischer Grenzen, Ländergrenzen, Eingrenzung, Ausgrenzung oder andere Grenzen sein würden, die während den selbstbestimmten und meist selbstorganisierten Phasen zum Lernen anregen, blieb völlig offen. Natürlich brachte die internationale Teilnehmerschar auch ihre ganz eigenen Erfahrungen zum Thema Grenze mit. So entstand beispielsweise eine sehr beeindruckende Collage zur Sichtweise auf die deutschdeutsche Grenze aus der Perspektive von Ost- und Westdeutschland. Eine Gruppe Studentinnen machte Erfahrungen mit den Sprachbarrieren eines Schweizer Kindergartenkindes, seiner Mundart und ihrem süddeutschen Dialekt. Daraus entwickelten sich interessante Gespräche über die schweizerisch-deutsche Grenzregion, ihre Grenzgänger im Bildungswesen und die Unterschiede der vermeintlich gleichen Kulturen.

\subsection{Einstieg: Ideen sammeln, Fragen finden und faszinieren}

\subsubsection{Schreibgespräch}

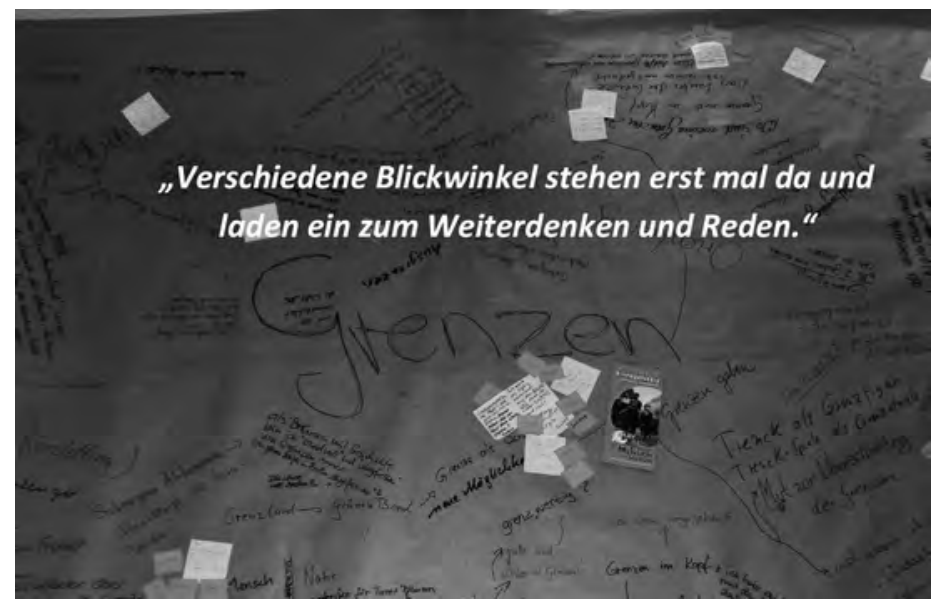

Abb. 2: Schreibgespräch „Grenzen“ 
Nach der allgemeinen Vorstellung der Tagung, des Ablaufs und der Organisatoren bekamen die Teilnehmer die erste Möglichkeit sich mit dem Thema "Grenze“ auseinanderzusetzen. Durch ein Schreibgespräch schafft man eine besonders konzentrierte Arbeitsatmosphäre, da die Teilnehmer ihre Gedanken schriftlich formulieren, was häufig präziser ist als der mündliche Austausch (Reich 2002). Zudem sind die Ergebnisse auch zu einem späteren Zeitpunkt einsehbar und können bei der Reflexion wertvolle Anhaltspunkte geben. Beim Schreibgespräch kamen erste eigene und gemeinsame Ideen zu Tage, die ohne eine bewusst von außen angeleitete Inspiration an das Präkonzept der jeweiligen Teilnehmer anknüpften. Während des Schreibgesprächs kommunizierten die Teilnehmer ausschließlich schriftlich miteinander und konnten dabei auf das Geschriebene Bezug nehmen. So zeigten sich erste gemeinsame Interessen, die oftmals zur Bildung von Forschergruppen führten. Zum Schluss entstand eine Art Mindmap, auf der sich jeder Teilnehmer wiederfinden konnte und erste thematische Fragen entstanden. Mit diesen Gedanken im Kopf hatten die Teilnehmer die Möglichkeit das Museum zu besichtigen, welches diverse Objekte und Ausstellungsstücke zum Thema Grenze zeigte.

Das Konzept des Museums sah die Reduktion auf wenige, sich vermeintlich widersprechende Objekte vor. Die Schwierigkeit bestand darin, in der Auswahl und Kombination der Objekte, den Installationen noch genügend Raum für freie Interpretationen zu lassen und somit Lernwege noch nicht vorweg zu nehmen. Worte und Textbausteine wurden aber aus oben genanntem Grund nicht verwendet, im Zusammenspiel mit den Objekten ergaben sich die Irritationen. Das Museum entstand mit der Absicht die Teilnehmer zum Staunen einzuladen, zum Denken anzuregen und den gegenseitigen Austausch über einzelne Exponate zu ermöglichen. Durch die Irritationen sollten die gängigen Vorstellungen zum Thema Grenze aufgebrochen und neue Zugänge sowie Herangehensweisen an die Thematik ermöglicht werden (vgl. Hameyer 2011). 


\subsection{Museum - Raum für Interpretation}

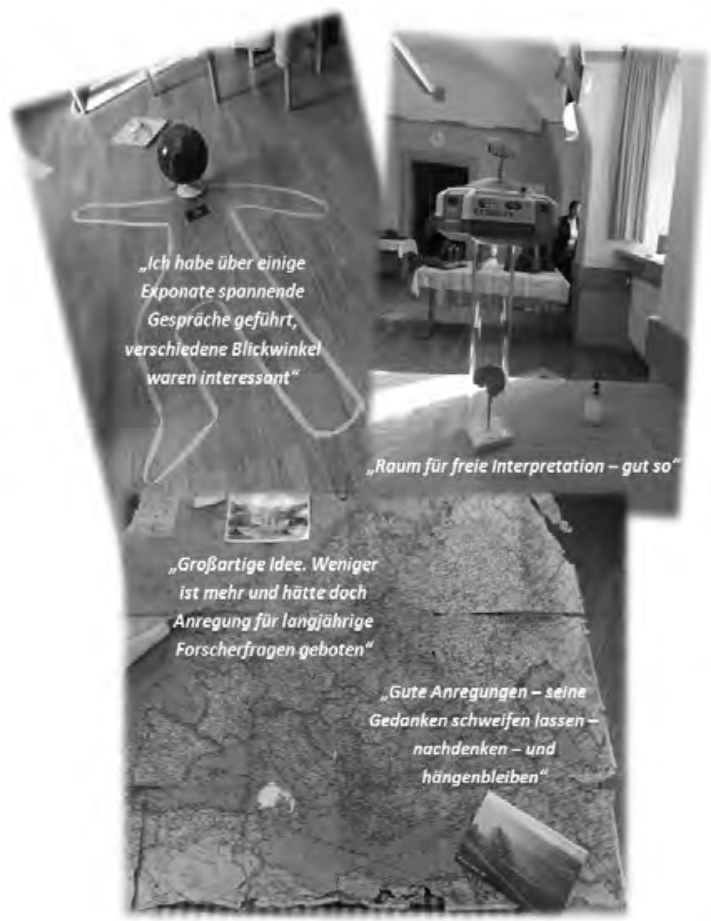

Abb. 3: Exponate im Museum

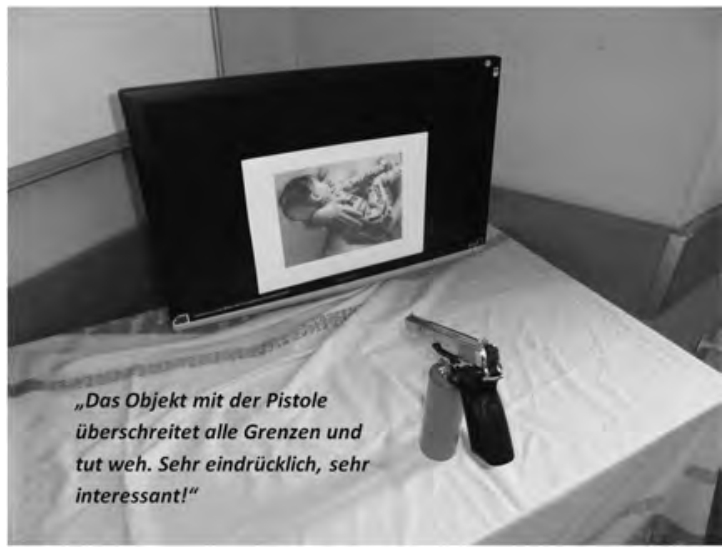

Abb. 4: Irritation im Museum 


\subsection{Die eigene Frage suchen}

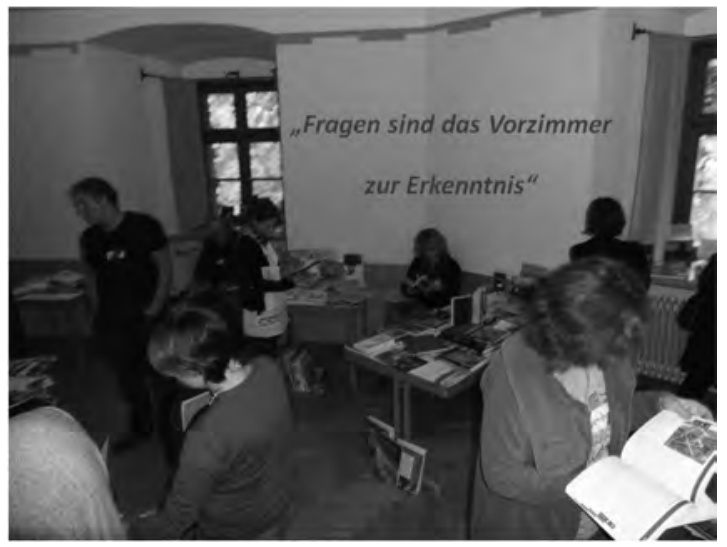

Abb. 5: Die Suche nach der eigenen Frage

Nach den Impulsen aus dem Schreibgespräch und dem Museum konnten sich die Teilnehmer ihren eigenen Fragestellungen zuwenden und diese entwickeln. Die Bibliothek, eine lose Sammlung von Büchern und Medien, ermöglichte eine erste Recherche zu verschiedenen Themen und fungierte darüber hinaus als Impulsgeber für die eigenen Fragen. Sie war in einem separaten Raum untergebracht. Sie wurde erst nach einer gewissen Zeit geöffnet, nachdem alle Teilnehmer im Museum gewesen waren, um die primäre Themenfindung durch die Medien nicht zu stark zu beeinflussen. Die Bibliothek hatte die Aufgabe, weitere Impulse zu bisherigen Fragen oder Fachwissen zum Thema bereit zu stellen. Wichtig erschien es den Organisatoren, deutlich zu machen, dass es nicht eine explizite Frage sein musste, die entstehen sollte, es konnte auch an einer eigenen Thematik gearbeitet werden. So stand im geleiteten Lerntagebuch der Tagung bewusst auch nicht alleinig der Titel „meine Forscherfrage“ sondern „meine Projektidee“. Dies wurde vor allem gewählt, um den Stress, eine gute Forscherfrage zu finden, besonders für die Neuen zu mindern und individuelle Herangehensweisen an ein Thema zuzulassen (vgl. DKJS 2013). Das Thema Grenze erschien zu Beginn sehr vielschichtig und philosophisch. Dies und unter anderem die Tatsache, dass nicht vorgegeben wurde, wie die Präsentation der Ergebnisse am Ende der Tagung aussehen sollte, stellten einige Teilnehmer vor eine Herausforderung. Gerade zu Beginn der Arbeitsphase wirkten einige Teilnehmer erschlagen von der Vielzahl der Möglichkeiten, die ihnen offen standen.

Als dritter Inspirations- und Arbeitsraum wurde die Werkstatt mit diversen Werk-, Bastel- und Verbrauchsmaterialien geöffnet. Während der gesamten Erarbeitungsphase standen die Bibliothek und die Werkstatt den Teilnehmern zur Verfügung. Auf Anfrage wurde zusätzlich benötigtes Material von den Organisatoren besorgt. 


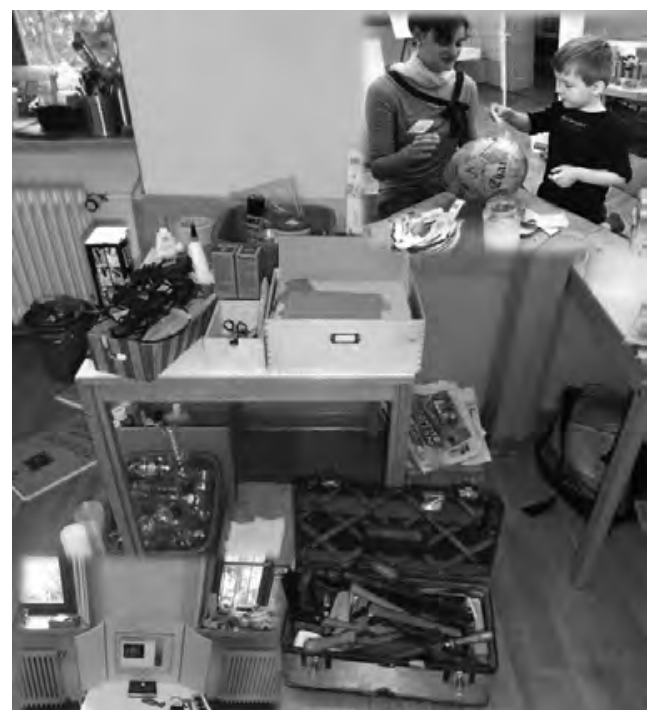

Abb. 6: Werkstatt der Möglichkeiten

\subsection{Lernbegleitung}

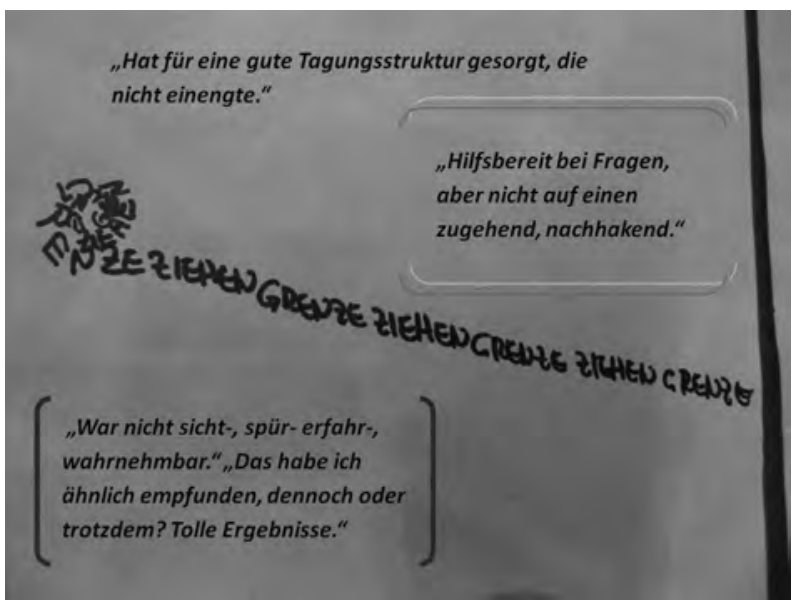

Abb. 7: Wahrnehmung der Lernbegleitung

Eine wichtige Rolle in jedem Lernprozess in einer Lernwerkstatt spielt die Lernbegleitung, über die im Verlauf der Tagung hitzig und produktiv diskutiert wurde. Für die Organisatoren, die in diesem Setting auch die Lernbegleiter waren, sollte die Lernbegleitung nicht aufdringlich sein. Ziel war, die Lernenden in erster Linie 
selbstständig arbeiten lassen. Es bestand die Möglichkeit für regelmäßige Treffen mit dem Lernbegleiter-Team, was vor allem von den teilnehmenden Studierenden angenommen wurde. In diesen Treffen wurden Fragen rund um die allgemeine Lernwerkstattarbeit gestellt, die Unsicherheit thematisiert, sich auf sein eigenes Thema einzulassen, ohne dass etwas von außen vorgegeben wurde. Zur Lernbegleitung gehörte auch eine große Pinnwand, auf der die Teilnehmer die Möglichkeit hatten, ihre Stimmungen auszudrücken, zum Beispiel durch die Auswahl von bereitgestellten Postkarten, die zum momentanen Stimmungsbild der Teilnehmer passten oder einfach durch das Aufschreiben des bisherigen Lernverlaufs. Ein regelmäßiger Austausch der Lernbegleiter über die Prozesse der Teilnehmer fand in kleiner Runde jeden Tag statt. In Kombination mit informellen Gesprächen, zum Beispiel während des gemeinsamen Abendessens, wussten die Lernbegleiter, auf wen sie zugehen konnten. Der Frage, ob eine Lernbegleitung, die nicht spürbar ist, auch bedeutet, dass sie nicht anwesend ist, mussten sich die Lernbegleiter im Verlauf der Tagung stellen. Dies kam besonders in der abschließenden Tagungsreflexion zum Ausdruck. Offensichtlich wurde die Lernbegleitung von den Teilnehmenden sehr unterschiedlich wahrgenommen. Von den einen Teilnehmern geschätzt und als Begleitung nach Bedarf wahrgenommen, wünschten sich andere mehr spürbare und öffentliche Begleitung z.B. in Form eines regelmäßigen Plenums mit kollegialer Lernbegleitung. Das wirft die Frage auf, inwieweit eine Pinnwandreflexion die allabendliche Plenumsrunde ersetzen oder ergänzen kann, damit sich jeder Lernende beteiligt und wahrgenommen fühlt, aber im Gegenzug derjenige, der sich lieber nicht äußern will und diese Zeit anders nutzen möchte, nicht auf seinem Lernweg gestört wird. „Gute Idee, da nicht jeder am Ende des Tages gezwungen wird, künstlich ein Statement abzugeben!“ kontra „Wäre schön gewesen, auch darüber zu sprechen“. Wie kann hier auf die unterschiedlichen Bedürfnisse zwischen den Teilnehmern in Zukunft eingegangen werden? Wie komme ich diesen unterschiedlichen Bedürfnissen bei meinen Schülern oder Studenten nach? Was lege ich als Lernbegleiter als Methode in diesem Setting fest, und welche Überlegungen dazu kommuniziere ich im Voraus? Diese in dieser Tagung ausprobierte zurückhaltende, beobachtende Art der Lernbegleitung zeigte eindrücklich, wie unterschiedlich die Bedürfnisse auch bei Erwachsenen sind und dass es so viele Lernwege wie Lernende gibt. 


\subsection{Prozessdokumentation, Lerntagebuch}

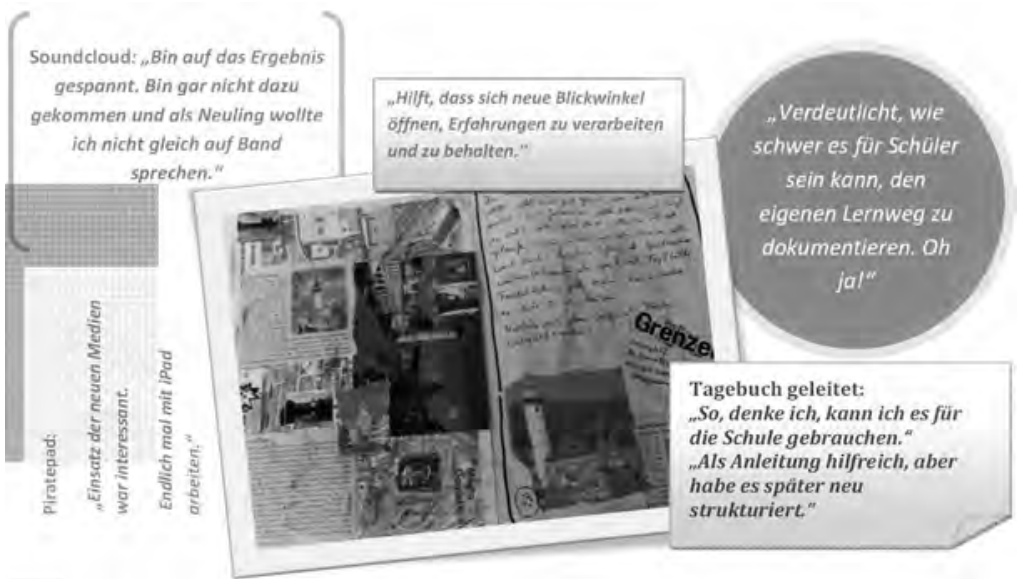

Abb. 8: Das Lernen dokumentieren

Wie bereits angedeutet, wurden bei der Lernwegdokumentation vielfältige und vorzugsweise neue Medien eingesetzt. Auf der Tagung wurde zwischen öffentlicher und privater Dokumentation des Lernwegs unterschieden. Die öffentlichen Dokumentationen beinhalteten die eben beschriebenen Pinnwände mit einem Stimmungsbarometer, die Anwendung einer Sprachaufzeichnung und des PiratePads als Austauschplattform, bei dem mehrere Akteure gleichzeitig an einer Aufzeichnung arbeiten können, beispielsweise als Projektdokumentation einer Arbeitsgruppe. Die Soundcloud wurde als mögliches akustisches Medium im Rahmen der Projektidee „Sprache und Kultur verbinden“ eingesetzt, indem mit ihr Interviews von Grundschulkindern aus Waldmünchen aufgenommen und bearbeitet wurden. Wer Interesse hatte, konnte sich die auf die Soundcloud aufgenommenen Statements der anderen Teilnehmer anschauen und -hören sowie diese kommentieren.

Die private Dokumentation des Lernwegs war ausschließlich den Lernenden zugänglich und wurde sowohl in einer instruierten als auch in einer offenen Form in DIN A4 und DIN A5 bereitgestellt. Dabei war das offene Lerntagebuch einzig eine Sammlung einheitlich gestalteter Blätter, ohne explizite Aufforderung zum Schreiben, während das instruierte, geführte Tagebuch seinem Besitzer Fragen stellte, die diesen zum Nachdenken über seinen eigenen Lernprozess anregen sollten. So wurden zum Beispiel die Fragen „Wann und wo tauchten Schwierigkeiten auf? Wie kam es dazu? Was mache ich damit? Was nehme ich mit?" gestellt. Das geführte Lerntagebuch wurde vermehrt als Anregung für den eigenen Unterricht bezeichnet und die meisten wichen, laut ihren Äußerungen, von der vorgegebenen Linie ab. Auch hier stellt sich die Frage, wie viel Instruktion nötig ist. Wo engt 
sie ein und wo schafft sie Wegweiser oder vom Lernenden gewünschte Grenzen im Lernprozess?

\subsection{Angebote zum Entdecken}

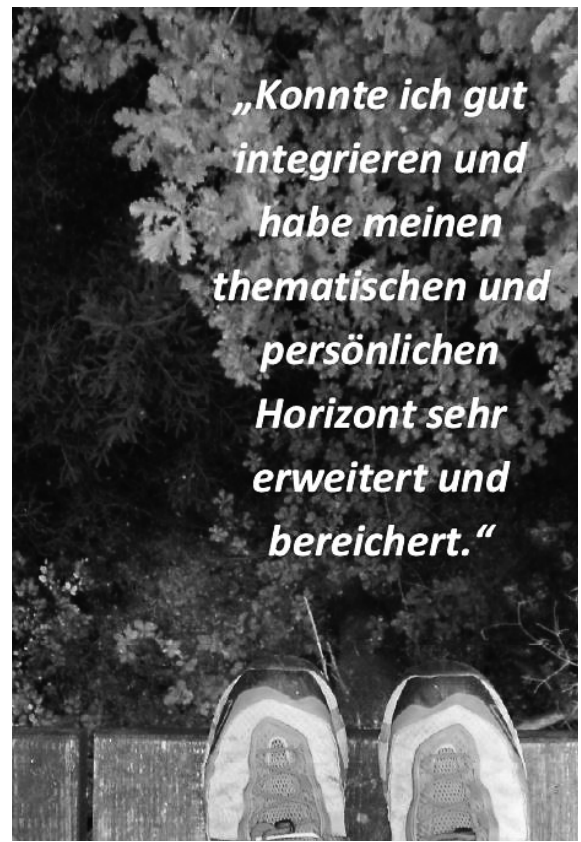

Abb. 9: Grenzerfahrung?

Während der Tagung hatten die Teilnehmer die Möglichkeit, ihren Horizont durch diverse Angebote in der Umgebung zu erweitern, die von den Organisatoren ausgesucht und organisiert wurden. So konnte zum Beispiel ein Hochseilgarten und ein Niederseilgarten besucht werden, in denen die Teilnehmer ihre persönlichen psychischen und physischen Grenzen austesten konnten. Außerdem hatten sie die Möglichkeit an einem Wünschelruten-Lehrpfad ihr übersinnliches Gespür unter Beweis zu stellen oder beim Eisvogelsteig, eingekleidet in eine Wathose und Sicherheitsgurt, einen Klettersteig im Fluss zu bewältigen. Letztendlich ging es bei den Angeboten immer wieder um das Thema Selbsterfahrung, die eigene Grenze auszuloten, um schließlich in der Reflexion das Erlebte einzugliedern in die eigene Vorstellungswelt und das eigene Projekt. Einige Teilnehmer bezeichneten diese Erfahrungen auch als ihren sehr persönlichen Lernweg, den sie im Vergleich zu ihrer Forscheridee nicht der Gruppe präsentierten, wohl aber die persönlich gemachten Erfahrungen in ihr Projekt einfließen ließen. Außer den bestehenden Angeboten organisierten die Teilnehmer selber einige 
Unternehmungen in der Nähe der Jugendbildungsstätte, wie zum Beispiel einen Grenzspaziergang entlang der deutsch-tschechischen Grenze.

\subsection{Lernwege und Produkte präsentieren}

Nach zwei Tagen intensiver Arbeitsphase hatten die Teilnehmer die Möglichkeit ihre Arbeitsergebnisse vorzustellen. Die Art und Weise, wie der Arbeitsprozess präsentiert werden sollte, blieb den Teilnehmern dabei frei überlassen, was unter den Teilnehmenden zu einiger Skepsis führte, inwiefern sinnvolle Ergebnisse entstehen würden. Um den Druck vom Lernprozess zu nehmen, wurde immer wieder betont, dass kein Endprodukt präsentiert werden müsse, es durften begonnene Forschungsideen ebenso wie entstandene Produkte und Ideen vorgestellt werden. Letztendlich wurde zum Ende der Freiarbeitsphase dennoch wieder von einem fest geplanten Präsentationsrahmen gesprochen, um allen Projekten gleichsam Anerkennung zu geben und auch wieder den Druck, der im Nachhinein oftmals als zu gering empfunden wurde, zu erhöhen, um alle Teilnehmenden zu motivieren etwas von ihrem Forschungsprojekt zu präsentieren. Vergleichbar mit der Entstehung des Museums zu Beginn der Tagung entstand eine Ausstellung, die von den Teilnehmern arrangiert wurde und zum Verweilen an den verschiedenen Objekten und zum gemeinsamen Austausch einlud. Dabei waren es die unterschiedlichen Zugänge und Themen der Teilnehmer, die dieser Ausstellung ihren Charakter verliehen. Man konnte deutlich erkennen, wie vielfältig die Vorstellungen zum Thema Grenze sein können und welche kreativen Ausstellungsstücke hervorgebracht wurden.

\subsection{Reflexion}

Was bleibt von der Lernwerkstätten-Tagung in Waldmünchen? Das Experiment einer jüngeren Gruppe von Organisatoren in der Lernwerkstätten-Community, eine Tagung zu gestalten, kann als gelungen gelten. Auch die Organisatoren gingen immer einen Weg an der Grenze zwischen einer wahrnehmbaren und einer versteckten Instruktion. Die neuen Methoden wurden zumeist positiv angenommen und wohlwollend in Diskussionen eingebracht. So stellt sich bei dem „tollen Versuch ein neues Medium nutzbar zu machen“ (schriftlicher Kommentar einer Teilnehmerin im PiratePad), also bei der Verwendung von Soundcloud, PiratePad und co. dennoch die Frage: Muss alles konserviert werden? Und was fängt man danach mit der Fülle an Material an? Auch bei der Präsentationsform gab es durchaus unterschiedliche Wahrnehmungen, ebenso verschieden wie das heterogene Teilnehmerfeld. Beispielsweise sind einige Darstellungsformen wie etwa ein Theaterstück, Tanz oder Gedicht weggefallen, was die Frage aufwirft, ob das entstandene Museumsformat bei der Präsentation der Arbeiten der Teilnehmer dadurch entstand, dass schon der Einstieg in das Thema damit begonnen wurde, ob es von den Teilnehmern selbst kam oder ob die Thematik zu dieser Darstel- 
lungsform beitrug. Hätte die abschließende Vorstellung der Forschungsprojekte in einer Vorstellungsrunde gemündet, wäre den oben genannten Darstellungsformen möglicherweise mehr Raum zugekommen als bei der Präsentation nach dem Museumscharakter, der seinerseits bestimmte Präsentationsformen bevorzugt. Nach einem abschließenden Schreibgespräch zu den oben vorgestellten Themen fand im Anschluss ein Plenum statt, indem jede und jeder noch einmal die Möglichkeit hatte, Wichtiges anzusprechen. Besonders angeregt wurde die Thematik rund um die Lernbegleitung, vor allem in Bezug auf die Wahrnehmbarkeit der Lernbegleitung diskutiert. Die Organisatoren wagten sich an die schmale Grenze der Lernbegleitung zwischen einer sehr engen, wahrnehmbaren Begleitung der Lernenden und einer in dieser Tagung eher unmerklichen und im Hintergrund ablaufenden Lernbegleitung. Es wurde deutlich, dass die Rolle der Organisatoren von den Teilnehmern nicht gleich gesetzt wurde mit der der Lernbegleitung. Daher muss man sich für künftige Treffen überlegen, ob eine Trennung von Organisation und Lernbegleitung sinnvoll ist oder wie man die Lernbegleiter kennzeichnen kann. Bei einer Lernbegleitung, die die TeilnehmerInnen auch als solche wahrnehmen, würden sich die meisten mehr als Lernende gewürdigt fühlen als es bei der Tagung der Fall war. Der Austausch der Lernbegleiter über die Lernprozesse der Lernenden, ohne konkrete Äußerungen derer, wirft die Frage auf, inwieweit dies vor dem Hintergrund einer Lernwerkstätten-Tagung von Erwachsenen ethisch vertretbar ist. Wo legt man also die Grenze der Lernbegleitung auf der Achse merkliche versus unmerkliche Instruktion? Wie viel Raum man in Zukunft der Rolle des Lernbegleiters geben möchte, und zwar nicht nur im Lernprozess selbst, sondern vielmehr auf der Metaebene, ist eine der Fragen, die es bei zukünftigen Tagungen zu diskutieren gilt. Eine tägliche Zusammenkunft der Lernenden und der Lernbegleitenden bietet sich an, in der jeder äußern kann, an was er gerade arbeitet, wo er möglicherweise feststeckt und insbesondere, wie er die Lernbegleitung erlebt hat, was er sich von ihr wünscht. Durch diese kontinuierliche Reflexion kann es gelingen ein gemeinsames Verständnis zu entwickeln, wie präsent eine Lernbegleitung sein kann, ohne dass sie als störend empfunden wird.

\section{Abschluss}

Blickt man auf die Tagung mit etwas Abstand zurück, wurde versucht eine Lernlandschaft zu kreieren, die über alle Disziplingrenzen hinaus wächst und eine Atmosphäre schafft, die ein möglichst freies Tätigsein ermöglicht. Der Wunsch mehr instruiert zu werden, auch in offenen Lernumgebungen, muss aufgegriffen werden und besonders im Schulalltag bedacht werden. So könnte es durch eine zu wenig greifbare Lernbegleitung und eine zu geringe Instruktion der Lernenden schnell zu einer Verunsicherung oder gar einer Überforderung kommen, was den Entdeckergeist und Tatendrang eines intrinsisch motivierten Lerners vehement einschränkt. Der Raum, in welchem man sich zwischen 
freiem und instruiertem Tätigsein und Fächerdisziplinen bewegt, kann letztendlich nur in gemeinsamer Reflexion abgesteckt werden. Denn gerade erst die Reflexion des Erfahrenen macht die Lernwerkstattarbeit sowohl für den Lernenden als auch die Lernbegleiter so wertvoll.

\section{Literatur}

Deutsche Kinder- und Jugendstiftung (DKJS) (2013): Thema finden, Fragen entwickeln. www.forschendes-lernen.net $\rightarrow$ Forschendes Lernen $\rightarrow$ Forschungsphasen (letzter Zugriff am 30.09.2013).

Deutsche Kinder- und Jugendstiftung (DKJS) (2011) (Hrsg.): Auf neuen Wegen. Die Lernkultur an Ganztagesschulen verändern. http://www.ganztaegig-lernen.de $\rightarrow$ Publikationen (letzter Zugriff am 30.09.2013).

Einstein, Albert (1986): Mein Weltbild. Ullstein: Frankfurt am Main.

Ernst, Karin (1988): Wie lernt man Offenen Unterricht. Erfahrungen aus der Lernwerkstatt der TU Berlin. In: Pädagogik, 40. Jg., Heft 6, 14-18.

Hagstedt, Herbert (2008): In Werkstätten lernen - wie Forscher arbeiten. http://www.forschendeslernen.net/files/eightytwenty/materialien/Hagstedt_In-Werkstaetten-lernen_14.pdf (letzter Zugriff am 30.09.2013).

Hameyer, Uwe (2011): Die Irritation der Gewohnheit. Wie Schulen und Schüler lernen und warum Umwege schneller sind. In: Deutsche Kinder- und Jugendstiftung (Hrsg.), 13-17.

Reich, Kersten (Hrsg.): Techniken. http://methodenpool.uni-koeln.de $\rightarrow$ Downloadcenter $\rightarrow$ Techniken (letzter Zugriff am 30.09.2013).

Wedekind, Hartmut (2006): Didaktische Räume - Lernwerkstätten - Orte einer basisorientierten Bildungsinnovation. In: Gruppe\&Spiel, 6.Jg., Heft 6, 9-12. http://www.wl-lang.de/Paedagogigsche\%20Leistungskultur/Lernbereich\%20Leist\%20Lernwerkstaetten\%20-\%20Didaktische\%20 Raeume.pdf (letzter Zugriff am 30.09.2013).

Weizsäcker, Richard von (2001): Polnisch-deutsche Verständigung nach dem Zweiten Weltkrieg. Mohr Siebeck: Tübingen. 